\title{
Effects of Perinatal Exposure to Phthalate/Adipate Esters on Hypothalamic Gene Expression and Sexual Behavior in Rats
}

\author{
Hwi-Cheul LEE ${ }^{1)}$, Keitaro YAMANOUCHI ${ }^{1)}$ and Masugi NISHIHARA ${ }^{1)}$ \\ ${ }^{1)}$ Department of Veterinary Physiology, Veterinary Medical Science, The University of Tokyo, \\ 1-1-1 Yayoi, Bunkyo-ku, Tokyo 113-8657, Japan
}

\begin{abstract}
Our previous research has identified the granulin (grn) and p130 genes as sex steroidregulated genes in the neonatal rat hypothalamus that might be involved in sexual differentiation of the brain. Since phthalate/adipate esters such as di-n-butyl phthalate (DBP), diisononyl phthalate (DINP), and di-2-ethylhexyl adipate (DEHA) are suspected to interfere with the endocrine system as environmental endocrine disruptors having estrogenic or antiandrogenic properties, these chemicals may affect sexual differentiation of the brain. The present study assessed the effects of perinatal exposure to DBP, DINP, and DEHA on grn and p130 mRNA expressions in the hypothalamus on postnatal day (PND) 7 and sexual behaviors after maturation in rats. Maternal rats were given a phytoestrogen-free diet containing different doses of DBP $(20,200,2,000$, and 10,000 ppm), DINP (40, $400,4,000$, and 20,000 ppm) and DEHA (480,2,400, and 12,000 ppm) from gestational day 15 to the day of weaning (PND 21). DBP and DINP exposure during the perinatal period resulted in an increase in hypothalamic grn and p130 mRNA levels in females and males, respectively, but DEHA exposure decreased expression levels of grn in males and p130 in females, although the effects were not dosedependent. After maturation, male rats that were exposed to several doses of DBP, DINP, and DEHA displayed decreased copulatory behavior. The lordosis quotient was decreased in females perinatally exposed to DBP, DINP, and DEHA at all the doses used. On the other hand, serum levels of LH and FSH in both sexes and the estrous cycles in females were not affected by the treatments. These results suggest that inappropriate expression of grn and/or p130 genes in the brains of male and female neonatal rats by perinatal exposure to these chemicals may exert permanent effects on the hypothalamus, thereby decreasing sexual behavior after maturation.
\end{abstract}

Key words: Adipate, Granulin, Hypothalamus, p130, Phthalate, Sexual behavior

(J. Reprod. Dev. 52: 343-352, 2006)

I n mammals, gonadal steroids play an essential role in the process of brain sexual differentiation during embryonic development and early postnatal life, which is known as the critical period. In this period, testosterone secreted from the testes in males acts to masculinize and defeminize the developing brain after conversion to estradiol by enzyme aromatase in the brain [1]. We

Accepted for publication: January 26, 2006

Published online: February 22, 2006

Correspondence: M. Nishihara (e-mail: amnishi@mail.ecc.utokyo.ac.jp) previously identified the granulin (grn) precursor gene [2] and p130 gene [3] as sex steroid-regulated genes in the rat hypothalamus during the critical period and suggested that these genes are involved in sexual differentiation of the rat brain [4-7]. According to our observations of the androgenand estrogen-dependent induction of grn and p130 genes and of the sexually different patterns of their gene expression in the hypothalamus, it is possible that these genes are good parameters for assessing the sex steroid properties of environmental endocrine disrupting chemicals (EDCs) in the 
neonatal brain [5].

EDCs include a number of environmental chemicals that interact with the endocrine system, often due to their activity as a hormonal mimic [8]. The majority of EDCs are recognized to bind estrogen receptors, which are members of a family of ligand-activated proteins that serve as transcriptional factors for a wide variety of cellular target genes, and act as agonists or antagonists of estrogen $[9,10]$. In recent years, phthalates have attracted much attention because several of these compounds are suspected of possess endocrine disrupting effects. Phthalate esters are used as plasticizers and are also found at low levels in foods, particularly fatty foods, such as dairy products, because they are fat-soluble [11, 12]. Din-butyl phthalate (DBP) initially attracted attention as a potential EDC because it was found to be a weak estrogen receptor agonist in some cell-based assays $[13,14]$. Several studies have suggested that DBP may disrupt the endocrine system and produce marked alterations during male reproductive organ development [15-17]. The effects are also closely associated with the antiandrogenic activity produced by DBP in developing fetuses and newborns [18, 19]. In regard to diisononyl phthalate (DINP), the most commonly reported findings in rodents are liver and kidney enlargement and increased levels of peroxisomal enzymes [20], and thus, DINP is recognized to have peroxisome proliferator properties. In utero exposure to DBP $[16,21]$, DINP [22], and di-2-ethylhexyl phthalate (DEHP) [21, 23] has been shown to disrupt differentiation of androgen-dependent tissues in male rat offspring. Since some phthalates have testis toxicity and antiandrogenic effects, alternative compounds, such as di-2-ethylhexyl adipate (DEHA), have replaced phthalates as plasticizers in flexible polyvinyl chloride (PVC) products [24]. Although DEHA is currently being evaluated as a potential substitute for some phthalates, due to the similarities in structure and metabolism of DINP, DEHP, and DEHA, similarities in their actions may also exist.

In the present study, we assessed the effects of perinatal exposure to DBP, DINP, and DEHA on several aspects of reproductive function in rats, especially their effects on sexual differentiation of the brain. To this end, maternal rats were given a diet containing these chemicals from gestational day 15 to the day of weaning, and anogenital distance (AGD) on postnatal day (PND) 1, serum sex-steroid hormone levels, and hypothalamic gene expression of grn and p130 on PND 7, and sexual behaviors, gonadotropin levels, and estrous cyclicity after maturation were examined.

\section{Materials and Methods}

\section{Test compounds}

DBP (CAS No. 84-74-2, purity $>98 \%$, Cat No. P0292) was purchased from Tokyo Kasei Kogyo Co., Ltd. (Tokyo, Japan). DINP (CAS No. 28553-120 , purity $>98 \%$, Cat No. 040-22805) and DEHA (CAS No. 103-23-1, purity $>99 \%$, Cat No. 027-13006) were purchased from Wako Pure Chemical Industries, Ltd. (Osaka, Japan).

\section{Animals and treatments}

This study followed the Guidelines for the Care and Use of Laboratory Animals of the Graduate School of Agricultural and Life Sciences of the University of Tokyo. Wistar-Imamichi rats (Imamichi Institute for Animal Reproduction, Ibaraki, Japan) that were approximately 8 weeks of age were kept in a room with controlled illumination (lights on 0500-1900) and temperature ( $23 \pm 1 \mathrm{C})$, and provided chow and water ad libitum. Animals were mated (mating confirmed by the presence of sperm in vaginal smears), and the day of mating was designated as gestational day (GD) 0 . The pregnant females were allowed to deliver pups naturally (day of birth=PND 0), and the litter size was adjusted to eight on PND 5. The dams were fed a pulverized soy-free diet [NIH-07-PLD (phytoestrogen-low diet); Oriental Yeast Co., Ltd., Tokyo, Japan] to reduce the effect of phytoestrogen. From GD 15 to the day of weaning (PND 21), the dams were provided a pulverized soy-free diet that contained 20, 200, 2,000, or 10,000 ppm of DBP, 40, $400,4,000$, or 20,000 ppm of DINP, or $480,2,400$, or $12,000 \mathrm{ppm}$ of DEHA. After weaning, all the animals were fed a conventional pellet diet (CRF-1; Oriental Yeast Co., Ltd., Tokyo, Japan).

\section{Experimental design}

On PND 1, body weight and AGD of pups were measured. On PND 7, pups were sacrificed by decapitation, and their brains were immediately removed. The entire hypothalamus, bordered 
anteriorly by the optic chiasma, laterally by the hypothalamic fissures, and posteriorly by the mammillary body, was then dissected out [6]. Hypothalamic blocks were frozen in liquid nitrogen and stored at $-80 \mathrm{C}$ until RNA isolation. Trunk blood was taken and centrifuged at 3,000 rpm for $15 \mathrm{~min}$ at $4 \mathrm{C}$. The serum was collected and stored at $-20 \mathrm{C}$ until assayed for serum testosterone and estradiol concentrations. After maturation, estrous cyclicity of females was examined by daily microscopic observation of vaginal smears during postnatal weeks (PNW) 8-9 and PNW 19-20. To determine the preovulatory gonadotropin surge, blood samples were collected from tail veins without anethesia at 1100 and $1600 \mathrm{~h}$ proestrus in female rats in PNW 20, and serum LH, FSH, and estradiol levels were measured. Blood samples were also taken from males in PNW 20 for measurement of serum $\mathrm{LH}, \mathrm{FSH}$, and testosterone levels.

In PNWs 20-21, copulatory behavior tests for both males and females were performed in a dark room at $1900 \mathrm{~h}$, and were recorded with a VCR system (DCR-TRV20 digital video camera, Sony, Tokyo, Japan). In each behavioral test, a sexually inexperienced male was placed in a plastic cage (40 $\times 22 \times 18 \mathrm{~cm}$ ). After a $2 \mathrm{~h}$ adaptation period, a highly estrous ovariectomized female, primed with a subcutaneous estradiol silastic tube $(70 \%$ cholesterol $/ 30 \% 17 \beta$-estradiol) for 5 days and injected with $1 \mathrm{mg}$ progesterone $6 \mathrm{~h}$ before the test, was introduced into the cage. Male sexual behavior was observed for $30 \mathrm{~min}$ after introduction of the female. The female was replaced by another similarly prepared female every $10 \mathrm{~min}$ to eliminate the influence of partner affinity. The following standard measures of male copulatory behavior were recorded: frequency of mount, intromission, and ejaculation during the $30 \mathrm{~min}$; and interval between first ejaculation and subsequent mount (post-ejaculation interval, PEI). Regarding the frequency of mount and intromission, when the male ejaculated during the $30 \mathrm{~min}$ experimental period, the number of mounts and intromissions from the time of female introduction to the first ejaculation was converted into the number per 30 min. As for females, the lordosis reflex was examined on the day of proestrus by sexually experienced vasectomized-males. The lordosis quotient (LQ) was recorded as the number of lordosis reflexes $/ 10$ mounts by males $\times 100(\%)$.

\section{Real-time quantitative RT-PCR}

Total RNA was isolated from the hypothalamus using TRIzol reagent (Invitrogen, CA, USA) according to the manufacturer's protocol. One microgram of total RNA was reverse-transcribed into cDNA using a GeneAmp RNA PCR Kit (Applied Biosystems, NJ, USA) in a reaction volume of $20 \mu \mathrm{l}$ according to the manufacturer's protocol. RT-PCR was performed on a LightCycler (Roche Diagnostics GmbH, Mannhaim, Germany) using LightCycler FastStart DNA Master SYBR Green I (Roche Diagnostics GmbH) according to the manufacturer's protocol. Primer sets were used for rat grn (forward primer, 5'AGTTCGAATGTCCTGACTCCGCCA-3'; reverse primer, $5^{\prime}$-AAGCCACTGCCCTGTTGGTCCTTT$3^{\prime}$ ), rat p130 (forward primer, 5'GTGTAAGTGCTGCCTCGGGCACAG-3'; reverse primer, 5'-CGGGAACATGACCTGAGTATCTGTTC-3') and rat 40S ribosomal protein S29 (RPS29) (forward primer, 5'-TGAAGGCAAGATGGGTCACCAGCAGC-3'; reverse primer, 5'-CAGGGTAGACAGTTGGTTTCATTGGG-3'). To construct a relative standard curve for each gene, PCR amplification was performed with template dilutions ranging from $10^{1}$ to $10^{3}$ copies. PCR amplification was performed under the following conditions: each cycle involved $15 \mathrm{~s}$ at $95 \mathrm{C}$ for denaturing, $15 \mathrm{~s}$ at $60 \mathrm{C}$ for annealing, and $15 \mathrm{~s}$ at 72 $\mathrm{C}$ for extension with 40 cycles. The cDNA was diluted one hundred-fold prior to PCR amplification.

\section{Hormone assays}

Serum concentrations of testosterone and estradiol were measured by testosterone and estradiol enzyme-immunoassays (EIA) kits (Cayman Chemical, Ann Arbor, MI, USA), respectively, according to the manufacturer's protocol. Serum concentrations of LH and FSH were measured by rat LH and FSH EIA systems (Amersham Pharmacia Biotech, Buckinghamshire, UK), respectively, according to the manufacturer's protocol.

\section{Statistical analysis}

Statistical analyses were conducted using StatView (version J5, Abacus Concepts, Inc.). Oneway analysis of variance and Dunnett's test were used to determine differences between the treated and control groups. Differences were considered 
statistically significant at $\mathrm{P}<0.05$.

\section{Results}

Effects on body weight and AGD on PND 1

All the dams used delivered live pups. There were no significant differences in the number and sex ratio of pups per litter at birth among the groups. On PND 1, the body weight of the control males was significantly higher than the control females (Fig. 1A). In the DBP, DINP, and DEHAexposed neonatal rats, body weights were significantly decreased compared with those of the control pups of the corresponding sexes, with the exception of the groups given $20 \mathrm{ppm}$ of DBP for both sexes and $200 \mathrm{ppm}$ of DBP for females. As shown in Fig. 1B, AGD in male neonates was decreased in a dose-dependent manner by maternal exposure to these chemicals. AGD in the 2,400 ppm and 12,000 ppm DEHA-exposed female neonates was also decreased. However, when AGD was normalized by the cube root of the body weight, exposure to DBP did not affect AGD in male neonates, although DINP at all doses used and the higher doses (2,400 and 12,000 ppm) of DEHA still decreased AGD in males (Fig. 1C). In female neonates, an increase in AGD was observed in DBP- and DINP-exposed animals at the highest doses, and the inhibitory effects of DEHA were no longer discernible.

\section{Effects on serum sex steroid levels and hypothalamic} gene expression on PND 7

In the control group on PND 7, serum testosterone levels were significantly higher in males than females (Fig. 2A), while there were no differences in serum estradiol levels between the sexes (Fig. 2B). Perinatal exposure to DBP, DINP, and DEHA did not affect the serum concentrations of testosterone and estradiol in either sex, with the exception that 2,000 ppm of DBP and $40 \mathrm{ppm}$ of DINP significantly decreased estradiol levels in female pups. On PND 7, hypothalamic expression of grn mRNA was higher in males (Fig. 3A), and that of p130 mRNA was higher in females (Fig. 3B). The expression of grn gene in female pups was increased by higher doses (2,000 and 10,000 ppm) of DBP and all the doses, except for 4,000 ppm, of DINP, while that in male pups was decreased by 480 and $12,000 \mathrm{ppm}$ of DEHA. Hypothalamic
A

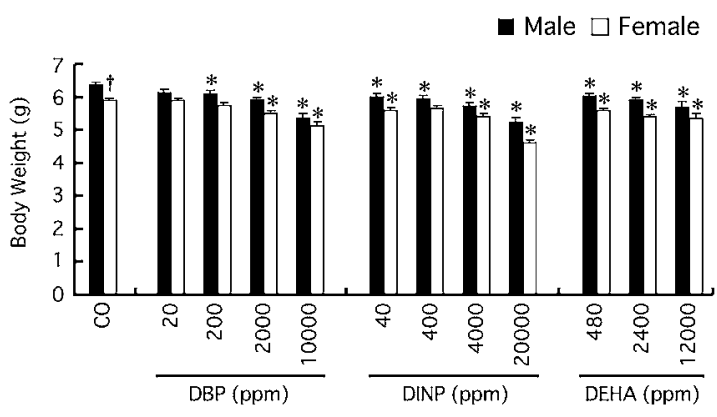

B

C
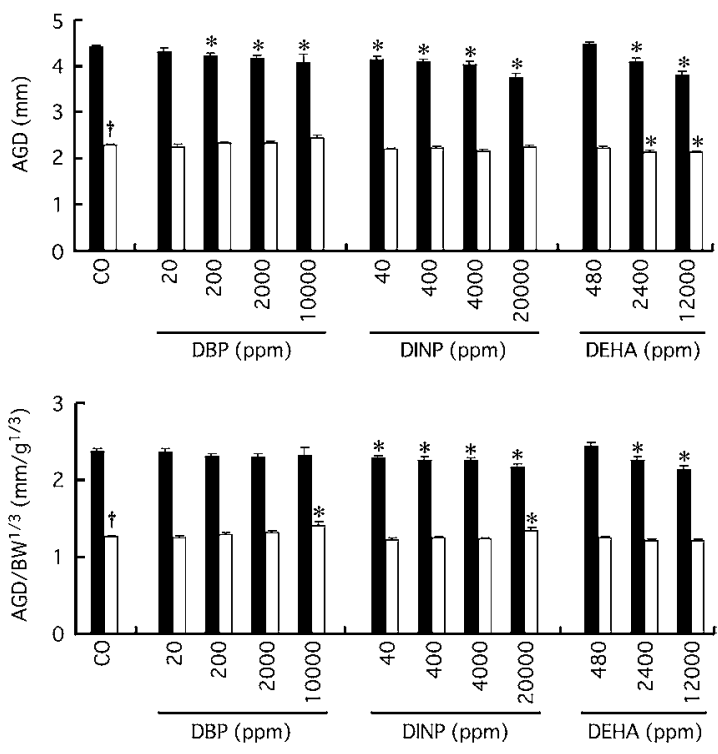

Fig. 1. Body weight (BW) (A), anogenital distance (AGD) (B), and AGD per cube root of BW ratio $(C)$ in male and female rats on PND 1. Each column and vertical bar represents the mean $\pm \mathrm{SE}(\mathrm{n}=16-47)$. $\mathrm{CO}$, control. *, $\mathrm{P}<0.05$ vs. $\mathrm{CO}$ of corresponding sex; ${ }^{+}, \mathrm{P}<0.05$ vs. male in $\mathrm{CO}$ group.

expression of p130 mRNA in males was increased by lower doses (20 and $200 \mathrm{ppm}$ ) of DBP and all the doses of DINP, whereas that of females was decreased by 480 and 2,400 ppm of DEHA.

\section{Effects on sexual behavior and serum gonadotropin levels after maturation}

Figure 4 shows the number of sexual behaviors in male rats examined in PNW 20-21. All of the control rats showed normal copulatory behaviors. Although the numbers of mounts and intromissions in the $40 \mathrm{ppm}$ DINP- and $480 \mathrm{ppm}$ DEHA-exposed rats were significantly decreased, perinatal exposure to DINP and DEHA at other doses and DBP at any dose did not affect the 
A

B
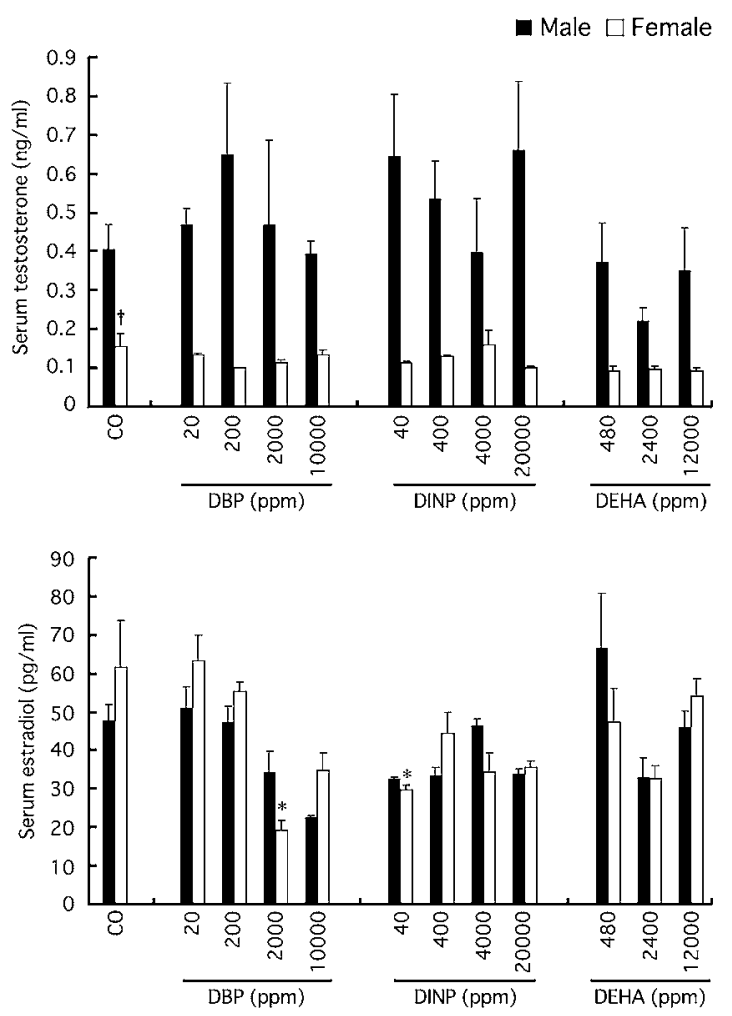

Fig. 2. Serum levels of testosterone (A) and estradiol (B) in male and female rats on PND 7. Each column and vertical bar represents the mean \pm SE $(n=5-7)$. CO, control. *, $\mathrm{P}<0.05$ vs. $\mathrm{CO}$ of corresponding sex; ${ }^{+}$, $\mathrm{P}<0.05$ vs. male in CO group.
A

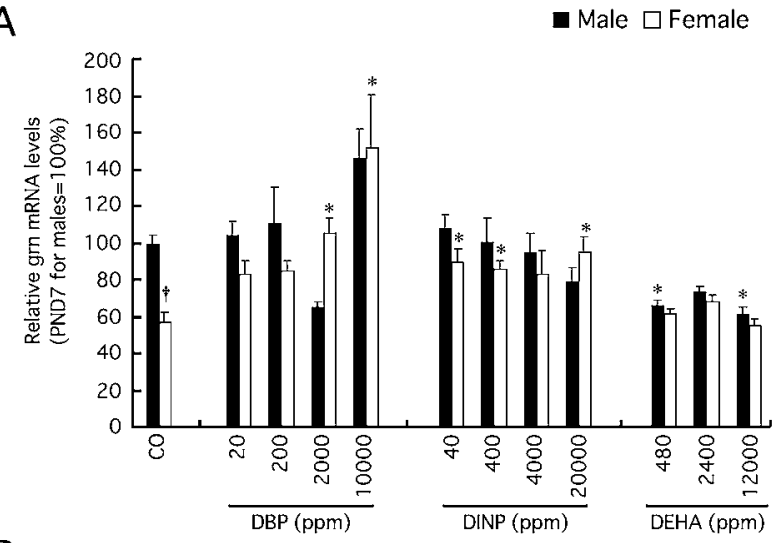

B

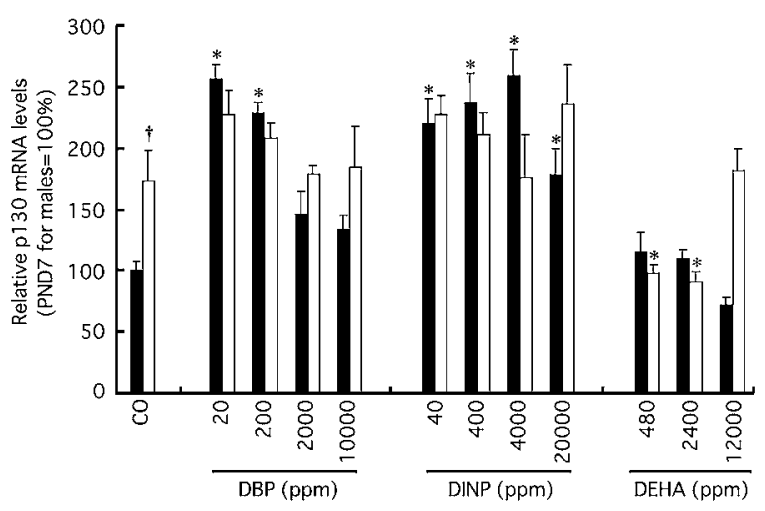

Fig. 3. Relative mRNA levels of grn (A) and p130 (B) in the hypothalamus of male and female rats on PND 7. Each value is normalized using RPS29. Each column and vertical bar represents the mean $\pm \mathrm{SE}(\mathrm{n}=5-7)$. CO, control. *, $\mathrm{P}<0.05$ vs. CO of corresponding sex; ${ }^{\dagger}$, $\mathrm{P}<0.05$ vs. male in $\mathrm{CO}$ group.

DEHA at any dose. The inhibitory effect of the chemicals on female copulatory behavior was dosedependent, except for DEHA. On the other hand, perinatal exposure to the chemicals did not affect serum levels of LH, FSH, and estradiol at either 1100 or $1600 \mathrm{~h}$ on the proestrous day in PNW 20 (Fig. 7).

\section{Discussion}

Previous studies have shown that in utero exposure to some phthalates produces a malformation of the male reproductive tract including a decrease in AGD, probably by reducing fetal testicular testosterone production [25-27]. It has been proposed that the relationship between 

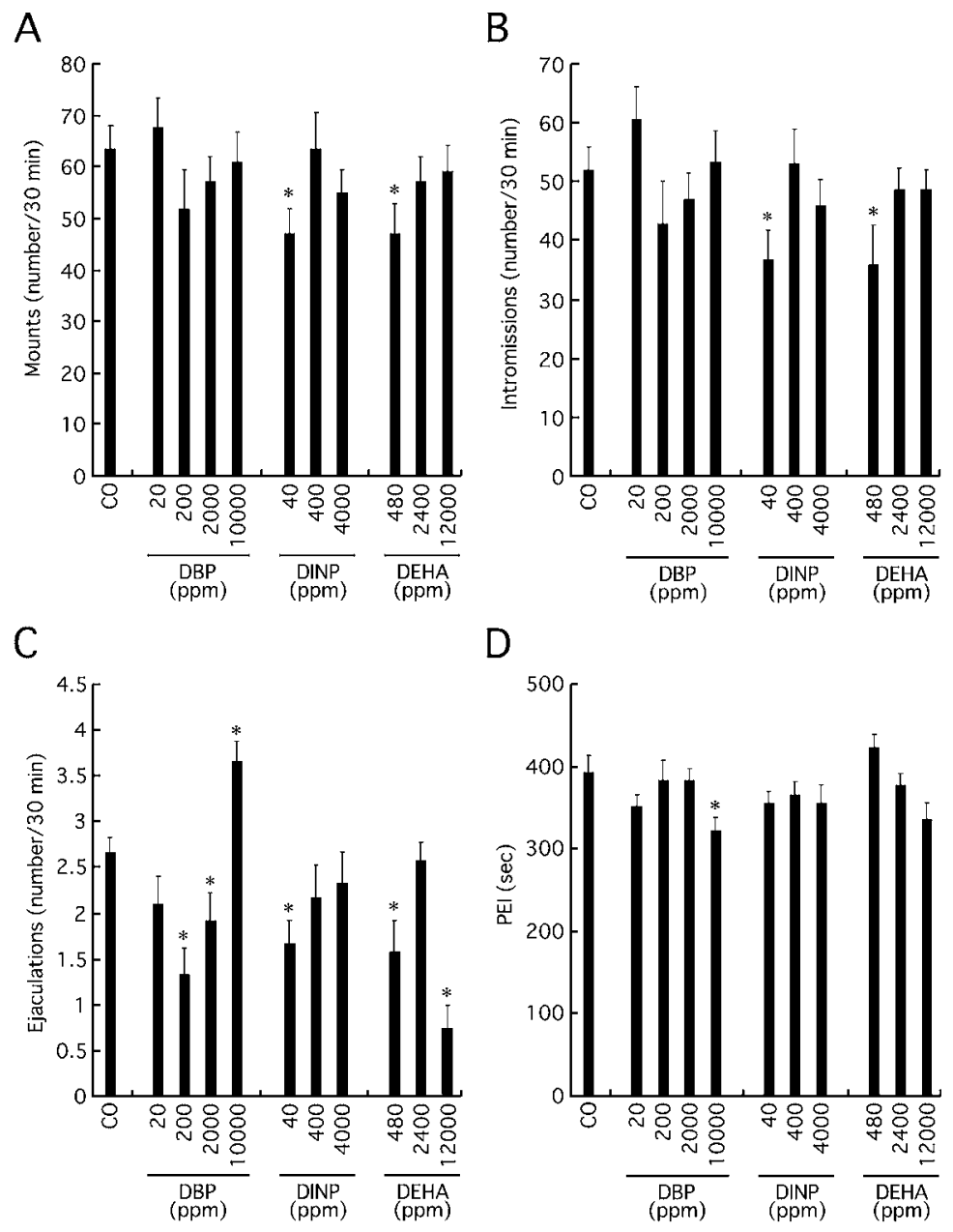

Fig. 4. The frequency of mount (A), intromission (B), ejaculation (C), and post-ejaculation interval (PEI) (D) in male rats in PNW 20. Each column and vertical bar represents the mean $\pm \mathrm{SE}(\mathrm{n}=$ 6-12). CO, control. *, $\mathrm{P}<0.05$ vs. CO.

AGD and body weight should be more properly evaluated using the cube root of the body weight $[28,29]$. In the present study, perinatal exposure to DINP and DEHA indeed decreased AGD in a dosedependent manner, even after normalization by the cube root of the body weight in male neonates, but the effect of DBP was no longer discernible. Since AGD is commonly regarded to be a measure of androgen-sensitive development in rodents [30], the antiandrogenic effect of DINP and DEHA appears to be more potent than that of DBP, at least under the present experimental conditions. Since there was no substantial change in serum testosterone and estradiol levels on PND 7, the inhibitory effect of the chemicals on fetal testicular testosterone production, if any, may be transient. Alternatively, DINP and DEHA might interact with androgen receptor as antagonists during the developmental period, and thus lead to a reduction in AGD. On the other hand, DBP and DINP at the highest dose used increased AGD normalized by body weight in female neonates, suggesting a large amount of these phthalates may also have weak androgenic properties.

In the present study, the gene expression of grn in the hypothalamus was higher in males and that of p130 was higher in females on PND 7, and this was consistent with our previous studies $[2,7]$. Both DBP and DINP increased grn mRNA levels in female neonates, although not dose-dependently, and did not affect the levels in males. Phthalates may affect the expression of these genes by directly acting on the hypothalamus because they did not affect the serum sex steroid levels on PND 7. Several in vitro studies have shown that phthalates, such as DBP and butylbenzylphthalate (BBeP), are capable of binding to estrogen receptor- $\alpha(E R \alpha)$, inducing $E R \alpha$-mediated gene expression, and enhancing the proliferation of MCF-7 human breast cancer cells, which express ER $\alpha[13,14,31,32]$. 
A

B
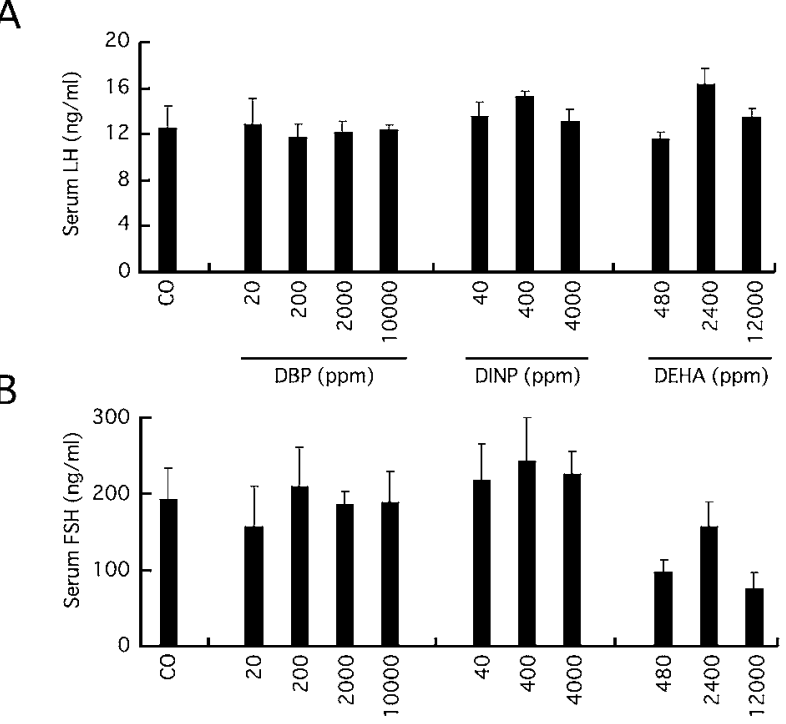

C

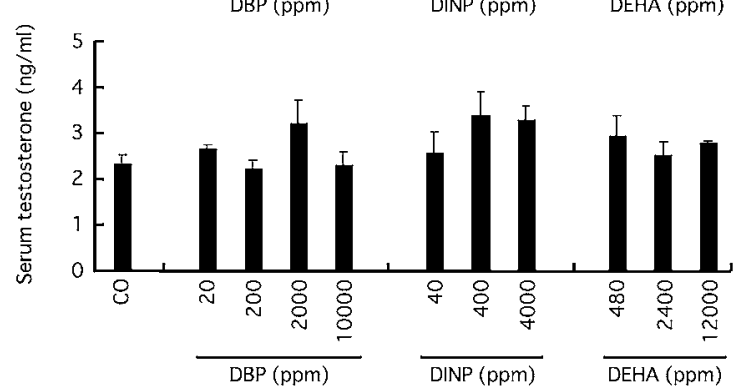

Fig. 5. Serum levels of LH (A), FSH (B), and testosterone (C) in male rats in PNW 20. Each column and vertical bar represents the mean $\pm S E(n=6-12)$.

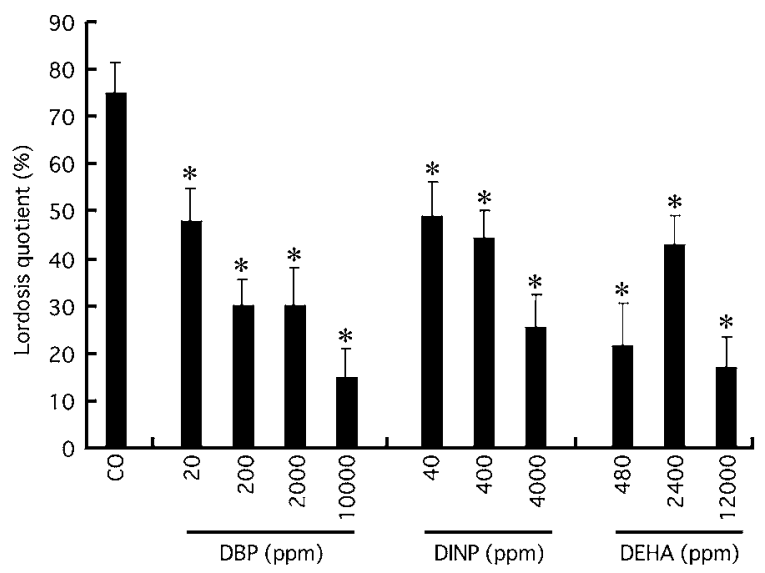

Fig. 6. The lordosis quotient of female rats in PNW 20. Each column and vertical bar represents the mean $\pm \mathrm{SE}(\mathrm{n}=$ 6-12). CO, control. *, $\mathrm{P}<0.05$ vs. CO.
A

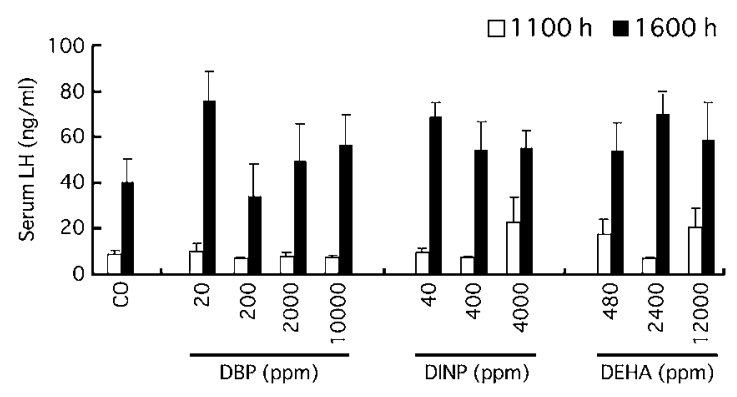

B

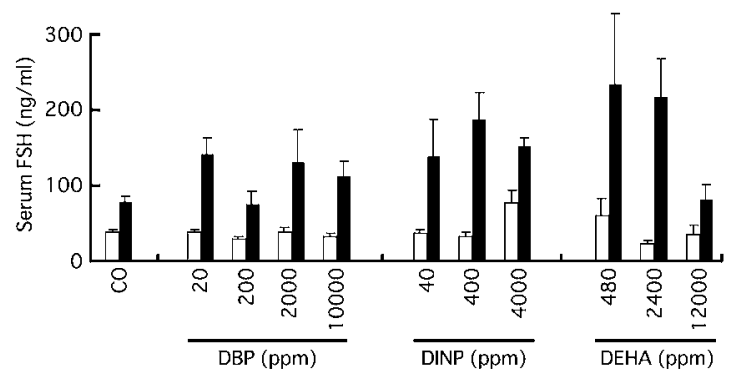

C

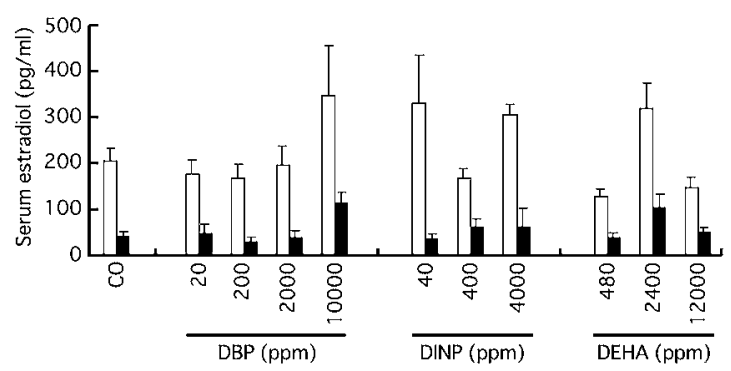

Fig. 7. Serum levels of LH (A), FSH (B), and estradiol (C) in female rats at 1100 and $1600 \mathrm{~h}$ on the day of proestrus in PNW 20. Each column and vertical bar represents the mean \pm SE $(n=6-12)$. CO, control.

Since we previously showed that androgen upregulates grn gene expression after conversion to estrogen [6], the increase in grn gene expression in the female hypothalamus may be due to the estrogenic properties of the phthalates. The reason why the effects of the chemicals were not dosedependent is currently unclear, but a similar nondose-dependent effect of DBP has been reported by others [33]. DBP and DINP increased p130 mRNA levels in only male neonates, though again not dose-dependently. The antiandrogenic properties of these compounds, especially in the case of DINP, may account for this increase in p130 gene expression. The effect of DEHA on gene expression was also sex specific, i.e., it decreased grn and p130 mRNA levels in males and females, respectively. This adipate might exert antiandrogenic effects on males and androgenic effects on females, but 
further studies are needed to clarify the presice mechanisms underlying the actions of phthalate/ adipate esters on sex steroid-dependent gene expression in the hypothalamus.

After maturation, serum levels of LH, FSH, and testosterone in the male rats were unchanged by perinatal exposure to DBP, DINP, and DEHA, indicating that these chemicals do not affect the endocrine system of hypothalamo-pituitarygonadal (HPG) axis in males. On the other hand, an inhibitory influence on male sexual behaviors was observed by perinatal exposure to these chemicals at several doses. The mount, intromission, and ejaculation frequencies were suppressed by the lowest doses of DINP and DEHA, and ejaculation was also suppressed by the highest dose of DEHA. The suppressive effect of DEHA on male sexual behavior might be due to the decrease in grn gene expression in the hypothalamus observed on PND 7 by DEHA, since a decrease in grn mRNA by infusion of an antisense oligodeoxynucleotide of grn gene into the third ventricle of male rats on PND 2 has been shown to result in a decrease in male sexual behavior after maturation [4]. Although the roles of p130 in the neonatal hypothalamus remain to be resolved, the suppressive effect of DINP on male sexual behavior might be related to an inappropriate increase in p130 gene expression in the neonatal hypothalamus. On the other hand, DBP at 200 and 2,000 ppm decreased ejaculation frequency, while it increased ejaculation frequency and shortened PEI at 10,000 ppm, without affecting the mount and intromission frequencies. As mentioned above, DBP at low doses increased p130 mRNA in the neonatal male hypothalamus, and at higher doses, increased grn mRNA in the neonatal female hypothalamus. Although not significantly, DBP at the highest dose also increased grn mRNA in the male hypothalamus. These changes in hypothalamic gene expression caused by perinatal exposure to DBP may account for its different effects on ejaculation after maturation. However, the possibility that other factors than grn and p130 are affected by perinatal exposure to the chemicals and involved in the decrease in sexual behavior in males after maturation cannot currently be ruled out.

In females, perinatal exposure to the chemicals used did not affect estrous cyclicity, serum estradiol levels, and gonadotropin surge on the day of proestrous. This is consistent with recent studies by Mylchreest et al. [26] and Masutomi et al. [33] who observed that perinatal exposure to DBP or DINP did not affect estrous cyclicity after maturation. Recurrence of the estrous cycle is regarded as one of the manifestations of the sexual dimorphic functions of the brain, and perinatal administration of sex steroids induces persistent vaginal estrus associated with the lack of estrous cyclicity in rats [35]. Reccurence of the estrous cycle depends on a female-specific function of the HPG axis, namely, induction of a preovulatory surge of gonadotropin-releasing hormone $(\mathrm{GnRH})$ and $\mathrm{LH}$, which is induced by the positive feedback action of estrogen. It is therefore probable that sexual differentiation of brain controlling gonadotropin secretion is not affected by perinatal exposure to DBP, DINP, and DEHA.

Contrary to the endocrine system, lordosis, the posture adopted by the female rat in response to a mounting male during mating, was decreased by perinatal exposure to all the chemicals used at any dose. Since DBP and DINP increased grn gene expression and DEHA decreased p130 gene expression in the female hypothalamus, although not all at the doses, changes in the expression of these genes may account for the suppression of female sexual behavior. If this is the case, grn and p130 may be involved in the processes of not only masculinization (increase in male-type sexual behavior), but also defeminization (decrease in female-type sexual behavior) of the rat brain during the neonatal period. The brain region responsible for inducing preovulatory GnRH surge is thought to be the medial preoptic area (MPOA) [36] and that responsible for inducing lordosis is thought to be the ventromedial nucleus of the hypothalamus (VMH) [37], where sexually dimorphic pattern of the synaptic organization exists [38, 39]. Since we have shown that both grn and p130 genes are mainly expressed in the medial basal hypothalamus, including the $\mathrm{VMH}$, the chemicals used in the present study may affect organization of the neuronal circuits in the VMH, but not the MPOA, by modulating expression of these genes during the processes of sexual differentiation of the brain.

In conclusion, the present study showed that inappropriate expression of grn and/or p130 genes in the hypothalamus of neonatal rats by perinatal exposure to DBP, DINP, and DEHA may result in a 
decrease in sexual behavior after maturation in both sexes, without affecting the endocrine system of the HPG axis. These chemicals may act directly on discrete regions of the hypothalamus that regulate sexual behaviors, but not gonadotropin secretion, thereby affecting sexual differentiation of the brain with a resulting decrease in sex-specific behavior in adulthood.

\section{Acknowledgements}

This work was supported in part by a Grant-inAid from the Ministry of Health, Labor and Welfare of Japan (Research for Food and Chemical Safety) and a Grant-in-Aid for Scientific Research from the Japan Society for the Promotion of Science.

\section{References}

1. MacLusky NJ, Naftolin F. Sexual differentiation of the central nervous system. Science 1981; 211: 12941302.

2. Suzuki M, Yoshida S, Nishihara M, Takahashi M. Identification of a sex steroid-inducible gene in the neonatal rat hypothalamus. Neurosci Lett 1998; 242: 127-130.

3. Yonehara K, Suzuki M, Nishihara M. Sex-related differences in gene expression in neonatal rat hypothalamus assessed by cDNA microarray analysis. Endocr J 2002; 49: 131-137.

4. Suzuki M, Bannai M, Matsumuro M, Furuhata $\mathbf{Y}$, Ikemura R, Kuranaga E, Kaneda Y, Nishihara M, Takahashi M. Suppression of copulatory behavior by intracerebroventricular infusion of antisense oligodeoxynucleotide of granulin in neonatal male rats. Physiol Behav 2000; 68: 707-713.

5. Suzuki M, Nishiahara M. Granulin precursor gene: a sex steroid-inducible gene involved in sexual differentiation of the rat brain. Mol Genet Metab 2002; 75: 31-37.

6. Suzuki M, Yonezawa T, Fujioka $H$, Matuamuro $M$, Nishihara M. Induction of granulin precursor gene expression by estrogen treatment in neonatal rat hypothalamus. Neurosci Lett 2001; 297: 199-202.

7. Yonehara K, Suzuki M, Yamanouchi K, Nishihara M. Androgen induces p130 mRNA expression in the neonatal rat hypothalamus. Neurosci Lett 2002; 334: 107-110.

8. Waldron AC, Naber EC. Importance of feed as an unavoidable source of pesticide contamination in poultry meat and eggs. 2. Residues in eggs and tissues. Poult Sci 1974; 53: 1428-1435.

9. Barlow S, Kavlock RJ, Moore JA, Schantz SL, Sheehan DM, Shuey DL, Lary JM. Teratology Society Public Affairs Committee position paper: developmental toxicity of endocrine disruptors to humans. Teratology 1999; 60: 365-375.

10. Colborn T, vom Saal FS, Soto AM. Developmental effects of endocrine-disrupting chemicals in wildlife and humans. Environ Health Perspect 1993; 101: 378384.

11. Page BD, Lacroix GM. The occurrence of phthalate ester and di-2-ethylhexyl adipate plasticizers in Canadian packaging and food sampled in 19851989: a survey. Food Addit Contam 1995; 12: 129-151.

12. Sharman M, Read WA, Castle L, Gilbert J. Levels of di-(2-ethylhexyl)phthalate and total phthalate esters in milk, cream, butter and cheese. Food Addit Contam 1994; 11: 375-385.

13. Harris CA, Henttu P, Parker MG, Sumpter JP. The estrogenic activity of phthalate esters in vitro. Environ Health Perspect 1997; 105: 802-811.

14. Jobling S, Reynolds T, White R, Parker MG, Sumpter JP. A variety of environmentally persistent chemicals, including some phthalate plasticizers, are weakly estrogenic. Environ Health Perspect 1995; 103: 582-587.

15. Gray LE, Ostby J, Furr J, Wolf CJ, Lambright C, Parks L, Veeramachaneni DN, Wilson V, Price $M$, Hotchkiss A, Orlando E, Guillette L. Effects of environmental antiandrogens on reproductive development in experimental animals. Hum Reprod Update 2001; 7: 248-264.

16. Mylchreest E, Sar M, Cattley RC, Foster PM. Disruption of androgen-regulated male reproductive development by di(n-butyl) phthalate during late gestation in rats is different from flutamide. Toxicol Appl Pharmacol 1999; 156: 81-95.

17. Mylchreest E, Wallace DG, Cattley RC, Foster PM. Dose-dependent alterations in androgen-regulated male reproductive development in rats exposed to Di(n-butyl) phthalate during late gestation. Toxicol Sci 2000; 55: 143-151.

18. Sharpe RM, Fisher JS, Millar MM, Jobling S, Sumpter JP. Gestational and lactational exposure of rats to xenoestrogens results in reduced testicular size and sperm production. Environ Health Perspect 1995; 103: 1136-1143.

19. Wine RN, Li LH, Barnes LH, Gulati DK, Chapin RE. Reproductive toxicity of di-n-butylphthalate in a continuous breeding protocol in Sprague-Dawley rats. Environ Health Perspect 1997; 105: 102-107.

20. Lington AW, Bird MG, Plutnick RT, Stubblefield WA, Scala RA. Chronic toxicity and carcinogenic evaluation of diisononyl phthalate in rats. Fundam 
Appl Toxicol 1997; 36: 79-89.

21. Gray LE Jr, Wolf C, Lambright C, Mann P, Price M, Cooper RL, Ostby J. Administration of potentially antiandrogenic pesticides (procymidone, linuron, iprodione, chlozolinate, p, $\mathrm{p}^{\prime}$-DDE, and ketoconazole) and toxic substances (dibutyl- and diethylhexyl phthalate, PCB 169, and ethane dimethane sulphonate) during sexual differentiation produces diverse profiles of reproductive malformations in the male rat. Toxicol Ind Health 1999; 15: 94-118.

22. Gray LE Jr, Ostby J, Furr J, Price M, Veeramachaneni DN, Parks L. Perinatal exposure to the phthalates DEHP, BBP, and DINP, but not DEP, DMP, or DOTP, alters sexual differentiation of the male rat. Toxicol Sci 2000; 58: 350-365.

23. Arcadi FA, Costa C, Imperatore C, Marchese A, Rapisarda A, Salemi M, Trimarchi GR, Costa G. Oral toxicity of bis(2-ethylhexyl) phthalate during pregnancy and suckling in the Long-Evans rat. Food Chem Toxicol 1998; 36: 963-970.

24. Petersen JH, Breindahl T. Specific migration of di(2-ethylhexyl)adipate (DEHA) from plasticized PVC film: results from an enforcement campaign. Food Addit Contam 1998; 15: 600-608.

25. Barlow NJ, Foster PM. Pathogenesis of male reproductive tract lesions from gestation through adulthood following in utero exposure to $\mathrm{Di}(\mathrm{n}$ butyl) phthalate. Toxicol Pathol 2003; 31: 397-410.

26. Mylchreest E, Cattley RC, Foster PM. Male reproductive tract malformations in rats following gestational and lactational exposure to Di(n-butyl) phthalate: an antiandrogenic mechanism? Toxicol Sci 1998; 43: 47-60.

27. Shultz VD, Phillips S, Sar M, Foster PM, Gaido KW. Altered gene profiles in fetal rat testes after in utero exposure to di-n-butyl phthalate. Toxicol Sci 2001; 64: 233-242.

28. Clark RL. Endpoints of reproductive system development. In: An Evaluation and Interpretation of Reproductive Endpoints for Human Health Risk Assessment. Washington DC: ILSI Press; 1999: 1027.

29. Wise LD, Vetter CM, Anderson CA, Antonello JM, Clark RL. Reversible effects of triamcinolone and lack of effects with aspirin or L-656,224 on external genitalia of male Sprague-Dawley rats exposed in utero. Teratology 1991; 44: 507-520.

30. Heinrichs WL. Current laboratory approaches for assessing female reproductive toxicity. In: Dixon RL (ed.), Reproductive Toxicology. New York: Reven Press; 1985: 95-108.

31. Andersen HR, Andersson AM, Arnold SF, Autrup $H$, Barfoed $M$, Beresford NA, Bjerregaard $P$, Christiansen LB, Gissel B, Hummel R, Jorgensen EB, Korsgaard B, Le Guevel $R$, Leffers $H$, McLachlan J, Moller A, Nielsen JB, Olea N, OlesKarasko A, Pakdel F, Pedersen KL, Perez P, Skakkeboek NE, Sonnenschein C, Soto AM, Sumpter JP, Thorpe SM, Grandjean P. Comparison of short-term estrogenicity tests for identification of hormone-disrupting chemicals. Environ Health Perspect 1999; 107 (Suppl 1): 89-108.

32. Zacharewski TR, Meek MD, Clemons JH, Wu ZF, Fielden MR, Matthews JB. Examination of the in vitro and in vivo estrogenic activities of eight commercial phthalate esters. Toxicol Sci 1998; 46: 282-293.

33. Masutomi N, Shibutani M, Takagi H, Uneyama C, Takahashi N, Hirose M. Impact of dietary exposure to methoxychlor, genistein, or diisononyl phthalate during the perinatal period on the development of the rat endocrine/reproductive systems in later life. Toxicology 2003; 192: 149-170.

34. Parks LG, Ostby JS, Lambright CR, Abbott BD, Klinefelter GR, Barlow NJ, Gray LE Jr. The plasticizer diethylhexyl phthalate induces malformations by decreasing fetal testosterone synthesis during sexual differentiation in the male rat. Toxicol Sci 2000; 58: 339-349.

35. Barraclough CA, Gorski RA. Evidence that the hypothalamus is responsible for androgen-induced sterility in the female rat. Endocrinology 1961; 68: 6879.

36. Caraty A, Fabre-Nys C, Delaleu B, Locatelli A, Bruneau G, Karsch FJ, Herbison A. Evidence that the mediobasal hypothalamus is the primary site of action of estradiol in inducing the preovulatory gonadotropin releasing hormone surge in the ewe. Endocrinology 1998; 139: 1752-1760.

37. Dornan WA, Akesson TR, Micevych PE. A substance P projection from the VMH to the dorsal midbrain central gray: implication for lordosis. Brain Res Bull 1990; 25: 791-796.

38. Matsumoto A, Arai Y. Sex difference in volume of the ventromedial nucleus of the hypothalamus in the rat. Endocrinol Jpn 1983; 30: 277-280.

39. Nishizuka $\mathbf{M}$, Arai $\mathbf{Y}$. Regional difference in sexually dimorphic synaptic organization of the medial amygdala. Exp Brain Res 1983; 49: 462-465. 